\title{
microRNA-25 promotes osteosarcoma cell proliferation by targeting the cell-cycle inhibitor p27
}

\author{
XIU-HUI WANG ${ }^{1}$, PAN CAI $^{1}$, MING-HUI WANG ${ }^{1}$ and ZHE WANG $^{2}$ \\ ${ }^{1}$ Department of Orthopaedics, Pudong New Area Zhoupu Hospital, Shanghai 201318; \\ ${ }^{2}$ Clinical Medical College, China Medical University, Shenyang, Liaoning 110001, P.R. China
}

Received November 15, 2013; Accepted April 24, 2014

DOI: $10.3892 / \mathrm{mmr} .2014 .2260$

\begin{abstract}
An increasing body of evidence indicates that microRNAs (miRNAs), a class of small non-coding RNAs, are often aberrantly expressed in human osteosarcoma. This study aimed to investigate the effects of miR-25 and to identify its potential target genes in osteosarcoma (OS) cells. First, the expression of miR-25 was detected by reverse transcription-quantitative polymerase chain reaction (RT-qPCR), which revealed a significant upregulation of miR-25 in osteosarcoma tissues compared to the adjacent healthy tissues. To investigate the role of miR-25 in osteosarcoma cell proliferation, the miR-25 precursor was next transfected into Saos-2 and U2OS cells. Overexpression of miR-25 promoted cell proliferation in vitro and tumor growth in a xenograft mouse model. In addition, our results revealed that the protein expression of p27, a cell-cycle inhibitor, is negatively regulated by miR-25. Restoring the p27 level in miR-25-overexpressing cells reversed the enhancing effect of miR-25 on cell proliferation. Therefore, miR-25 may act as an onco-miRNA in osteosarcoma, which provides new perspectives in cancer treatment strategies based on molecular targeting.
\end{abstract}

\section{Introduction}

microRNAs (miRNAs), a class of short non-coding RNA molecules, function as transcriptional or post-transcriptional regulators of gene expression $(1,2)$. Deregulation of miRNAs is tightly associated with human disorders, including obesity, cardiovascular diseases and tumorigenesis $(3,4)$. Recent studies have also shown that a number of miRNAs are upregulated or downregulated and play critical roles in osteosarcoma development $(5,6)$. For instance, miR-376c was shown to inhibit cell proliferation and invasion in osteosarcoma by targeting

Correspondence to: Dr Zhe Wang, Clinical Medical College, China Medical University, No. 92 Bei'er Road, Heping, Shenyang, Liaoning 110001, P.R. China

E-mail: zpyygkwxh@sina.cn; wang_1072111zhe@163.com

Key words: osteosarcoma, microRNA, miR-25, p27 transforming growth factor- $\alpha$ (7), whereas miR-221 induces osteosarcoma cell survival through enhancing the AKT signaling pathway (8).

Previous studies have shown that miR-25 expression and function is deregulated in several types of tumor (9-12). It was shown that miR-25 is consistently highly expressed in the serum of patients with breast cancer or hepatocellular carcinoma, indicating that this miR may be used as a biomarker in diagnosis and treatment $(9,10)$. In addition, the miR-25 level was significantly increased in human gastric cancer (GC) tissues and cell lines (11). Overexpression of miR-25 markedly enhanced cell proliferation, migration and invasion in GC cells, whereas inhibition of miR-25 caused a significant reduction in proliferation rates and a significant increase in apoptosis (11). However, miR-25 was found to be downregulated in human colon cancer tissues when compared to matched, non-neoplastic mucosa tissues (12). Functional studies revealed that restoration of the miR-25 expression inhibits cell proliferation and migration (12). By contrast, miR-25 inhibition promoted cell proliferation and migration (12). Therefore, miR-25 appears to act as either an onco-miRNA or a tumor suppressor in different types of cancer, and plays a crucial role in cancer biology. However, its biological functions in osteosarcoma remain unexplored to date.

\section{Materials and methods}

Cell culture and tissue samples. Osteosarcoma cell lines (Saos-2 and U2OS) were obtained from the American Type Culture Collection (Rockville, MD, USA). Cells were cultured in Gibco ${ }^{\circledR}$ RPMI-1640 (Thermo Fisher scientific, Inc., Beijing, China) supplemented with $10 \%$ Gibco $^{\circledR}$ fetal bovine serum (Thermo Fisher Scientific, Inc.). Tumor tissues and adjacent noncancerous normal tissues were collected from routine therapeutic surgery at the Department of Orthopaedics, Pudong New Area Zhoupu Hospital. A total of 25 samples (male; median age, 57 years; range, 48-65 years) were obtained with informed consent from patients with osteosarcoma, and the procedures were approved by the Institutional Review Board of the hospital. Subjects were excluded if they had other diseases, including biliary obstructive diseases, and acute or chronic virus hepatitis. The individuals with an alcohol consumption of $\geq 120 \mathrm{~g} /$ week for men at the time of the study or in the prior 6 months were also excluded from the study. 
Analysis of miRNA expression. miRNA from tissue samples and cell lines was harvested using the Ambion ${ }^{\circledR}$ mirVana miRNA Isolation kit (Thermo Fisher Scientific, Inc., Grand Island, NY, USA), following the manufacturer's instructions. All RNA samples were examined as to their concentration and purity. RNA purity was measured using the NanoDrop ND-1000 spectrophotometer (Thermo Fisher Scientific, Waltham, MA, USA). Based on the absorbance ratio at $260 / 280 \mathrm{~nm}$ $($ mean \pm standard deviation $=1.86 \pm 0.03)$, all RNA samples were pure and protein free. Expression of mature miRNAs was assessed with the Applied Biosystems ${ }^{\circledR}$ TaqMan ${ }^{\circledR}$ microRNA assay (Thermo Fisher Scientific, Inc.) with probes specific to the human hsa-miR25 (5'-aggcggagacuugggcaauug-3') and the small nuclear U6 snRNA, used as an internal control (5'-cauugaccauggacauacgacug-3'). The quantitative (q)PCR reaction was performed using a TaqMan Universal PCR Master mix on an Applied Biosystems ${ }^{\circledR}$ 7900HT Real-Time PCR system (all from Thermo Fisher Scientific, Inc.). Briefly, PCR conditions included an initial holding period at $95^{\circ} \mathrm{C}$ for $5 \mathrm{sec}$ and $60^{\circ} \mathrm{C}$ for $30 \mathrm{sec}$ for 45 cycles. Relative quantitation analysis of the gene expression data was conducted according to the $2^{-\Delta \Delta C \mathrm{t}}$ method.

Plasmid construction and transfection. To construct the miR-25 expression plasmid, the precursor sequence of the human miR-25 was cloned into Ambion ${ }^{\circledR}$ pSilencer $^{\mathrm{TM}}$, while the negative control (NC) plasmid contained a scrambled sequence (both from Thermo Fisher Scientific, Inc.). For transfection, Lipofectamine ${ }^{\circledR} 2000$ (Thermo Fisher Scientific, Inc.) was employed following the manufacturer's instructions.

Bromodeoxyuridine (BrdU) assay. To assess cell proliferation, an enzyme-linked immunosorbent assay (ELISA) was used, based on the incorporation of BrdU during DNA synthesis, (BrdU kit; Beyotime Institute of Biotechnology, Shanghai, China), following the manufacturer's protocols. All experiments were performed in triplicate. The absorbance of the samples at $450 \mathrm{~nm}\left(\mathrm{~A}_{450}\right)$ was measured on a SpectraMax 190 ELISA reader (Molecular Devices, Sunnyvale, CA, USA).

Western blot analysis. Cells were harvested and lysed with ice-cold lysis buffer containing $50 \mathrm{mM}$ Tris- $\mathrm{HCl}, \mathrm{pH} 6.8$, $100 \mathrm{mM}$ 2-Mercaptoethanol, $2 \% \mathrm{w} / \mathrm{v}$ sodium dodecyl sulfate (SDS) and $10 \%$ glycerol. Following centrifugation at $4^{\circ} \mathrm{C}$, proteins in the supernatants were quantified by a BCA quantification kit (Beyotime Institute of Biotechnology), separated by $10 \%$ SDS-polyacrylamide gel electrophoresis (PAGE), and transferred onto nitrocellulose membranes (Amersham Biosciences, Buckinghamshire, UK). Anti-p27 antibody (Cell Signaling Technology, Inc., Danvers, MA, USA) was used at 1:2,000 overnight at $4^{\circ} \mathrm{C}$, and anti-glyceraldehyde 3-phosphate dehydrogenase (GAPDH) antibody (Cell Signaling Technology, Inc.) was used at 1:5,000 overnight at $4^{\circ} \mathrm{C}$. The membranes were then incubated with the HRP-linked secondary antibodies (Cell Signaling Technology, Inc.). The signals were detected by SuperSignal West Pico Chemiluminescent Substrate kit (Pierce Biotechnology, Inc., Rockford, IL, USA) according to manufacturer's instructions. The images were visualized by a LAS-4000 Luminescent Image analyzer (Fujifilm, Tokyo, Japan). The p27 protein level was quantified using Quantity One software (Bio-Rad, Hercules, CA, USA) and was normalized to that of GAPDH.

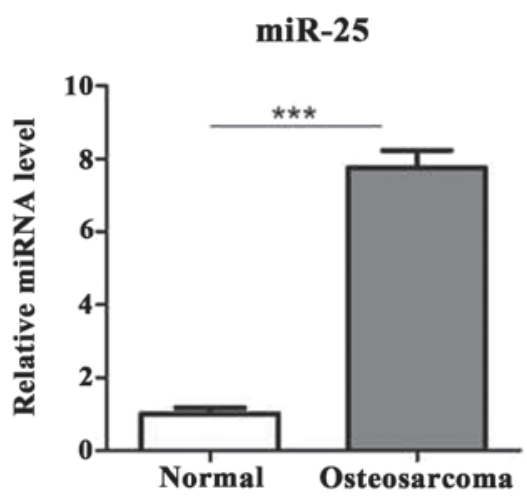

Figure 1. Expression level of miR-25 in osteosarcoma tissues. miR-25 expression was determined by reverse transcription-quantitative polymerase chain reaction assays in human osteosarcoma tissues and adjacent noncancerous tissues (normal). ${ }^{* * *} \mathrm{P}<0.001$.

Luciferase reporter assay. Total cDNA from Saos-2 cells was synthesized from total RNA using random hexamers with the Superscript III Reverse Transcriptase kit (Invitrogen, Carlsbad, CA, USA), according to the manufacturer's instructions. The reactions were incubated in a thermal cycler for $30 \mathrm{~min}$ at $16^{\circ} \mathrm{C}$, $30 \mathrm{~min}$ at $42^{\circ} \mathrm{C}, 5 \mathrm{~min}$ at $85^{\circ} \mathrm{C}$ and then held at $4^{\circ} \mathrm{C}$. The cDNA was used to amplify the $3^{\prime}$ untranslated region ( $3^{\prime}$ UTR) of $p 27$ by PCR. Mutations were introduced in potential miR-25-binding sites (5'-GCUAUUACGAAUACAUCCGUUAAC-3' was changed into 5'-GCUAUUACGAAUACAUCCGAAUUC-3') using the QuickChange ${ }^{\circledR}$ Site-Directed Mutagenesis kit (Stratagene, La Jolla, CA, USA). The pRL-SV40 vector (Promega Corp., Madison, WI, USA) carrying the Renilla luciferase gene was used as an internal control of transfection efficiency. Luciferase values were determined using the Dual-Luciferase ${ }^{\circledR}$ Reporter Assay system (Promega Corp.).

Tumor growth assay. Male BALB/c nude mice, 4 weeks-old, were purchased from the Shanghai Laboratory Animal Center (Shanghai, China). Saos-2 cells $\left(2 \times 10^{5}\right)$ were subcutaneously injected into the skin under the legs of the mice. The mice were observed for 5 weeks for tumor formation. Following sacrifice, the tumors were recovered and the wet weights of each tumor were measured.

Statistical analysis. Data were expressed as the mean \pm SEM from at least three independent repetitions of the experiment. Differences between groups were analyzed using Student's t-tests or one way analysis of variance (ANOVA). $\mathrm{P}<0.05$ was considered to indicate a statistically significant difference.

\section{Results}

The miR-25 expression level is increased in osteosarcoma tissues. First, in order to examine whether the miR-25 is differentially expressed in human osteosarcoma compared to noncancerous tissues, its expression level was determined using reverse transcription (RT)-qPCR in 25 pairs of human osteosarcoma tissues and pair-matched adjacent noncancerous tissues. The results demonstrated that the expression level of miR-25 was significantly increased in osteosarcoma tissues compared to the adjacent noncancerous tissues (Fig. 1). 
A

miR-25 (Saos-2 cells)

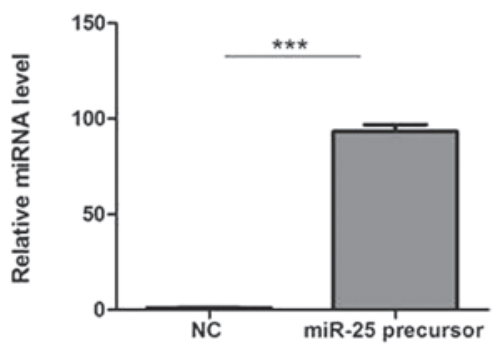

C

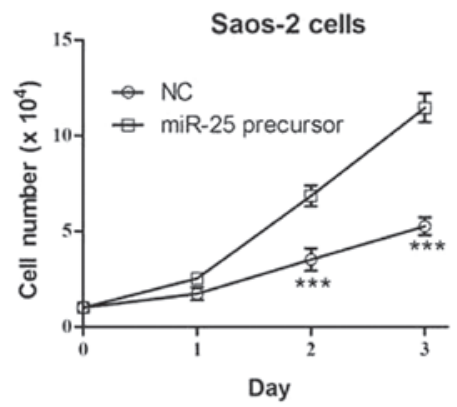

E

Saos-2 cells

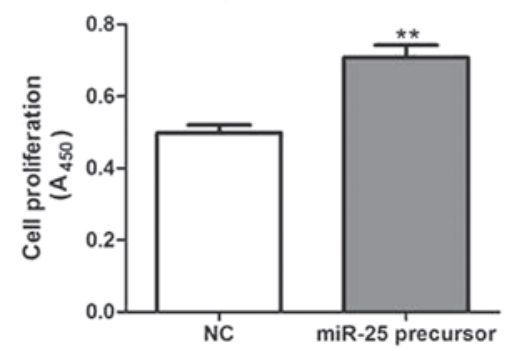

B

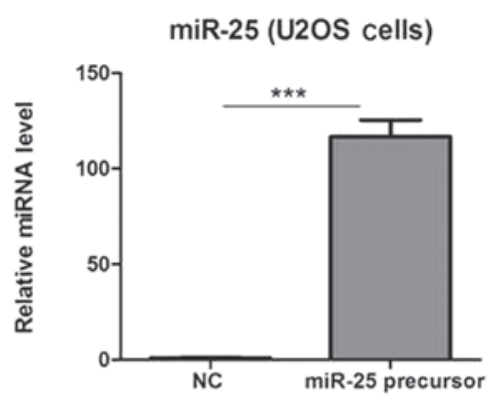

D

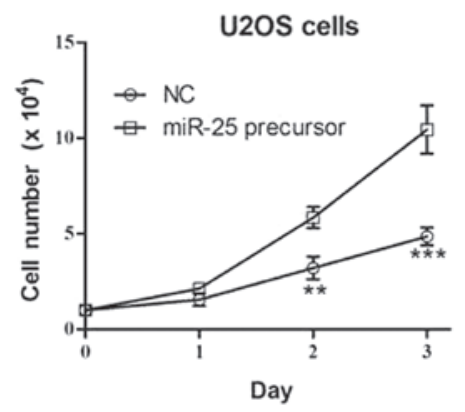

$\mathrm{F}$

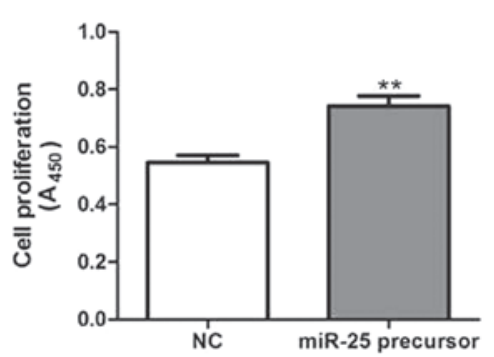

Figure 2. Overexpression of miR-25 promotes osteosarcoma cell proliferation. Expression of miR-25 was determined in (A) Saos-2 and (B) U2OS cells following transfection with the plasmid bearing the miR-25 precursor or the negative control (NC) plasmid. The growth curve of (C) Saos-2 and (D) U2OS cells following miR-25 precursor transfection as compared to that a transfection with NC. Cell proliferation was determined by the bromodeoxyuridine (BrdU) assay in (E) Saos-2 and (F) U2OS cells transfected with the miR-25 precursor-bearing plasmid or the NC. Absorption at $450 \mathrm{~nm}\left(\mathrm{~A}_{450}\right)$ was measured $24 \mathrm{~h}$ after the transfection. ${ }^{* *} \mathrm{P}<0.05 ;{ }^{* * *} \mathrm{P}<0.01$.

A

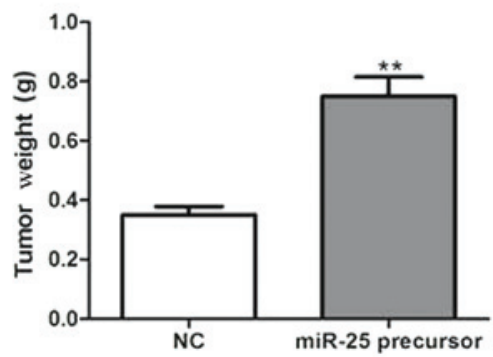

B

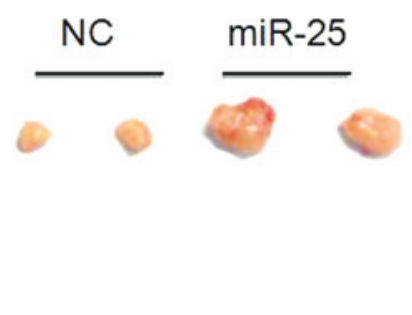

Figure 3. miR-25 promotes osteosarcoma cell proliferation in vivo. Saos-2 cells mock-transfected (NC) or stably transfected with miR-25 were injected into nude mice ( $n=6$ for each group), which were monitored for tumorigenesis. (A) Tumor weights and (B) representative pictures of tumors 4 weeks following the injection.

miR-25 overexpression promotes cell proliferation in vitro. In order to assess the effects of miR-25 on osteosarcoma cell growth, Saos-2 and U2OS cells were transfected with a plasmid bearing the miR-25 precursor or the NC plasmid, and cell growth was subsequently examined. Transfection with the miR-25 precursorbearing plasmid increased miR-25 expression compared to the $\mathrm{NC}$ transfection (Fig. 2A and B), and significantly increased the cell number and proliferation in both cell lines (Fig. 2C-F). 
A

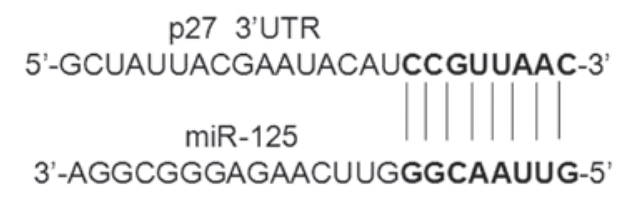

B

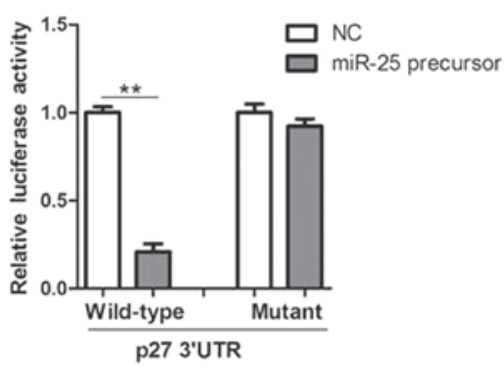

$\mathrm{F}$

Patient 1

Normal Osteosarcoma

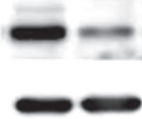

$\mathrm{C}$

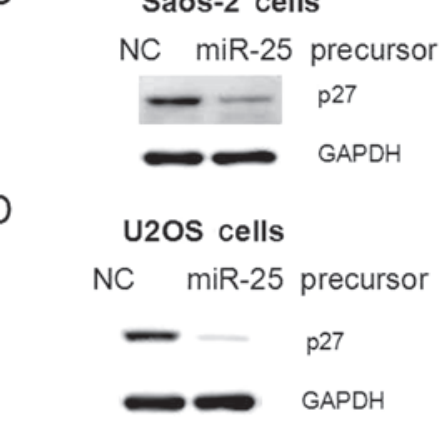

E

Tumors in nude mice

NC miR-25

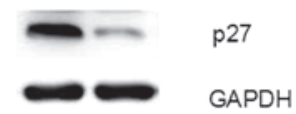

Patient 2

Normal Osteosarcoma

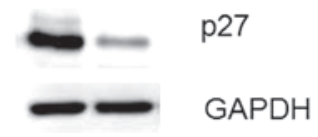

Figure 4. miR-25 negatively regulates p27 expression in osteosarcoma cells. (A) Predicted miR-25-binding sites (highlighted in bold) in the 3' untranslated region (3' UTR) of the human gene p27. (B) Luciferase reporter assays in Saos-2 cells. Cells were transfected with $200 \mathrm{ng}$ of the wild-type 3' UTR reporter or mutant constructs together with $20 \mathrm{nM}$ of the miR-25 precursor or a scrambled control. Representative western blots showing the relative expression of the protein p27 in (C) Saos-2 and (D) U2OS cells transfected with the miR-25 precursor or negative control (NC), (E) tumors from nude mice and (F) two patients with osteosarcoma. The glyceraldehyde 3-phosphate dehydrogenase (GAPDH) level was used as the loading control.

\section{A}

\section{Saos-2 cells}

NC miR-25 miR-25

EV EV P27

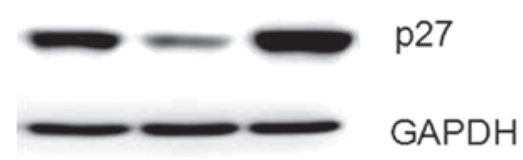

B

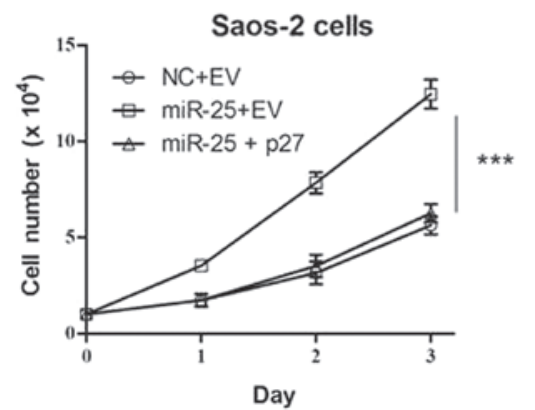

C

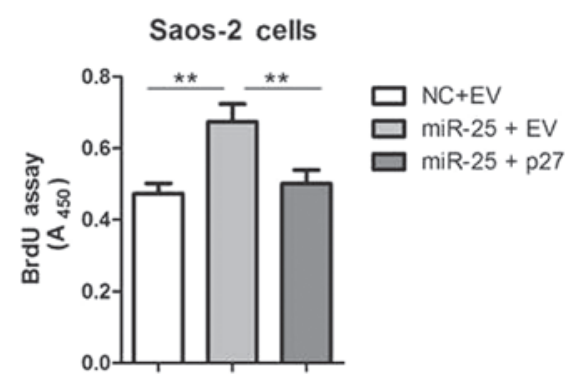

Figure 5. Re-introduction of p27 reverses the promoting effect of miR-25 on cell proliferation. (A) p27 protein expression was determined by western blot analysis in Saos-2 cells. Cells were pre-transfected with the miR-25 precursor or the negative control (NC) for $24 \mathrm{~h}$ and then transfected with expression plasmids for p27 or the empty vector (EV) for another $24 \mathrm{~h}$. (B) The growth curve and (C) cell proliferation rate was determined in Saos-2 cells. Absorption at $450 \mathrm{~nm}\left(\mathrm{~A}_{450}\right)$ was measured $30 \mathrm{~h}$. after the transfection with p27 or EV. 
miR-25 overexpression promotes cell proliferation in vivo. To study the role of miR-25 overexpression in tumorigenesis in vivo, we generated Saos-2 cells stably overexpressing miR-25 and injected the cells into a xenograft mouse model. miR-25 overexpression markedly promoted tumorigenesis in comparison with the NC, as evidenced by tumor weights and sizes (Fig. 3A and B).

miR-25 targets the p27 in osteosarcoma cells. Since miR-25 overexpression promoted cell proliferation and tumor growth, we examined the underlying mechanisms. Through a stringent bioinformatics approach (miRWalk software; http://www.umm. uni-heidelberg.de/apps/zmf/mirwalk/), we identified the gene $p 27$, encoding a cell-cycle inhibitor, as a candidate target of miR-25, since $p 27$ bears a potential miR-25-binding site (Fig. 4A). The 3' UTR of $p 27$ was cloned into a reporter luciferase system. When the reporter construct contained the $p 373^{\prime}$ UTR, overexpression of miR-25 led to a reduction in luciferase activity (Fig. 4B). By contrast, mutation of the conserved miR-25-binding motif abrogated the reduced luciferase expression (Fig. 4B). In addition, overexpression of miR-25 in osteosarcoma cells led to a reduction in the protein level of p27 (Fig. 4C and D). In addition, the p27 protein level was reduced in miR-25-overexpressing tumors and human osteosarcoma tissues (Fig. 4E and F), further supporting that $p 27$ may be a target of miR-25 in osteosarcoma cells.

p27 restoration attenuates the promoting effect of $m i R$ - 25 overexpression on cell proliferation. In order to confirm the functional connection between miR-25 and p27, Saos-2 cells were transfected with p27 expression plasmids following transfection with miR-25 (Fig. 5A). As shown in Fig. 5B and C, the re-introduction of p27 reversed miR-25-induced cell proliferation, indicating that the interaction between miR-25 and p27 is involved in this process. Taken together, our results suggest that the gene $p 27$ is an important target of miR-25 in osteosarcoma cells.

\section{Discussion}

In this study, we demonstrate that miR-25 expression is increased in osteosarcoma tissues. Overexpression of miR-25 by means of cell transfection promoted cell proliferation in Saos-2 and U2OS cells. Therefore, our study provided evidence, for the first time to the best of our knowledge, that miR-25 may act as an onco-miRNA, promoting the progression of osteosarcoma. It is notable that miR-25 was shown to enhance cell proliferation, migration and invasion in GC cells, but inhibit cell proliferation and migration in colon cancer cells $(11,12)$. Although the reasons for this inconsistence remain unexplored, we hypothesize that the biological functions of miR-25 in tumorigenesis may be cell- or tissue-specific. Therefore, the efficiency and safety associated with the use of miR-25 in gene therapy should be seriously considered in future studies.

Our study explored the mechanisms underlying the miR-25 effects, and revealed that $p 27$ maybe a target of miR-25 in osteosarcoma cells. $p 27$ encodes an enzyme inhibitor that belongs to the Cip/Kip family of cyclin-dependent kinase (CDK) inhibitor proteins (13). Through binding to the CDK 2 and CDK4 complexes, it prevents their activation, and controls the cell-cycle progression at the G1 phase $(14,15)$. Transcriptional or post-transcriptional downregulation of the gene has been observed in a number of human malignancies, including osteosarcoma $(16,17)$. For instance, $p 27$ was shown to be negatively regulated by miR-24, miR-200 and miR-222 in human cancer (18-20). Therefore, our results provide a novel mechanism for the downregulation of p27 in osteosarcoma.

Taken together, the key finding of the present study is that miR-25 can promote osteosarcoma cell proliferation in vitro and in vivo by targeting $p 27$, suggesting that miR-25 may be used as a molecular target for the treatment of osteosarcoma. However, the roles of this miRNA in osteosarcoma need to be further investigated.

\section{References}

1. Sun K and Lai EC: Adult-specific functions of animal microRNAs. Nat Rev Genet 14: 535-548, 2013.

2. van Kouwenhove M, Kedde M and Agami R: MicroRNA regulation by RNA-binding proteins and its implications for cancer. Nat Rev Cancer 11: 644-656, 2011.

3. Ameres SL and Zamore PD: Diversifying microRNA sequence and function. Nat Rev Mol Cell Biol 14: 475-488, 2013.

4. Kasinski AL and Slack FJ: Epigenetics and genetics. MicroRNAs en route to the clinic: progress in validating and targeting microRNAs for cancer therapy. Nat Rev Cancer 11: 849-864, 2011.

5. Liang W, Gao B, Fu P, Xu S, Qian Y and Fu Q: The miRNAs in the pathgenesis of osteosarcoma. Front Biosci (Landmark Ed) 18: 788-794, 2013

6. Cai CK, Zhao GY, Tian LY, et al: miR-15a and miR-16-1 downregulate CCND1 and induce apoptosis and cell cycle arrest in osteosarcoma. Oncol Rep 28: 1764-1770, 2012.

7. Jin Y, Peng D, Shen Y, et al: MicroRNA-376c inhibits cell proliferation and invasion in osteosarcoma by targeting to transforming growth factor-alpha. DNA Cell Biol 32: 302-309, 2013.

8. Zhao G, Cai C, Yang T, et al: MicroRNA-221 induces cell survival and cisplatin resistance through PI3K/Akt pathway in human osteosarcoma. PLoS One 8: e53906, 2013.

9. Li LM, Hu ZB, Zhou ZX, et al: Serum microRNA profiles serve as novel biomarkers for HBV infection and diagnosis of HBV-positive hepatocarcinoma. Cancer Res 70: 9798-9807, 2010.

10. Hu Z, Dong J, Wang LE, et al: Serum microRNA profiling and breast cancer risk: the use of miR-484/191 as endogenous controls. Carcinogenesis 33: 828-834, 2012

11. Zhao H, Wang Y, Yang L, Jiang R and Li W: MiR-25 promotes gastric cancer cells growth and motility by targeting RECK. Mol Cell Biochem 385: 207-213, 2014.

12. Li Q, Zou C, Zou C, et al: MicroRNA-25 functions as a potential tumor suppressor in colon cancer by targeting Smad7. Cancer Lett 335: 168-174, 2013.

13. Chu IM, Hengst L and Slingerland JM: The Cdk inhibitor $\mathrm{p} 27$ in human cancer: prognostic potential and relevance to anticancer therapy. Nat Rev Cancer 8: 253-267, 2008.

14. Frescas D and Pagano M: Deregulated proteolysis by the F-box proteins SKP2 and beta-TrCP: tipping the scales of cancer. Nat Rev Cancer 8: 438-449, 2008.

15. Mitrea DM, Yoon MK, Ou L and Kriwacki RW: Disorder-function relationships for the cell cycle regulatory proteins $\mathrm{p} 21$ and $\mathrm{p} 27$. Biol Chem 393: 259-274, 2012.

16. Kitagawa $\mathrm{K}$ and Kitagawa M: The SCF ubiquitin ligases involved in hematopoietic lineage. Curr Drug Targets 13: 1641-1648, 2012.

17. Maran A, Shogren KL, Benedikt M, Sarkar G, Turner RT and Yaszemski MJ: 2-methoxyestradiol-induced cell death in osteosarcoma cells is preceded by cell cycle arrest. J Cell Biochem 104: 1937-1945, 2008.

18. Giglio S, Cirombella R, Amodeo R, Portaro L, Lavra L and Vecchione A: MicroRNA miR-24 promotes cell proliferation by targeting the CDKs inhibitors p27Kip1 and p16INK4a. J Cell Physiol 228: 2015-2023, 2013.

19. Fu Y, Liu X, Zhou N, et al: MicroRNA-200b stimulates tumour growth in TGFBR2-null colorectal cancers by negatively regulating p27/kip1. J Cell Physiol 229: 772-782, 2014.

20. Kedde M, van Kouwenhove M, Zwart W, Oude Vrielink JA, Elkon R and Agami R: A Pumilio-induced RNA structure switch in p27-3' UTR controls miR-221 and miR-222 accessibility. Nat Cell Biol 12: 1014-1020, 2010. 